\title{
Indistinguishability of quantum states and rotation counting
}

\author{
Dmitri V. Averin ${ }^{1}$ and Christoph Bruder ${ }^{2}$ \\ ${ }^{1}$ Department of Physics and Astronomy, Stony Brook University, SUNY, Stony Brook, NY 11794-3800 \\ ${ }^{2}$ Department of Physics, University of Basel, Klingelbergstrasse 82, CH-4056 Basel, Switzerland
}

(Dated: July 23, 2018)

\begin{abstract}
We propose a quantum system in which the winding number of rotations of a particle around a ring can be monitored and emerges as a physical observable. We explicitly analyze the situation when, as a result of the monitoring of the winding number, the period of the orbital motion of the particle is extended to $n>1$ full rotations, which leads to changes in the energy spectrum and in all observable properties. In particular, we show that in this case, the usual magnetic flux period $\Phi_{0}=h / q$ of the Aharonov-Bohm effect is reduced to $\Phi_{0} / n$.
\end{abstract}

The properties of quantum systems depend crucially on whether nominally different points in configuration space are regarded as distinguishable or identical. As a basic example, consider a free particle on an infinite line. If points separated by a distance $L$ are regarded to be identical, its quantum-mechanical propagator will be radically modified and will coincide with that of a particle on a ring of circumference $L$ [1]. Typically, the question of the indistinguishability of different points appears for angular variables, e.g., the phase of the electromagnetic field in quantum optics 2 4 , the phase difference of superfluid condensates in the Josephson effect [5-9], or the rotation angle $\phi$ in real space. Considering for simplicity a planar rotation of one point particle, or a planar rotor, it seems natural that the states that correspond to the angles $\phi$ and $\phi+2 \pi$ which differ by one full rotation are identical. The resulting $2 \pi$-periodicity of the real-space rotations of a planar rotor manifests itself in a variety of properties of many physical systems, in particular, in the Aharonov-Bohm (AB) effect with period $h / q$ for particles of charge $q$, like Cooper pairs in superconducting rings 10, 11 or electrons in normal metal rings 12 14 .

Although the indistinguishability of states that differ by one full rotation in real space is typical, it is not absolute: in principle, the process of rotation can be observed, making angular states that differ by $2 \pi$ distinguishable. Such an observation will alter the quantum properties of the rotating system, which depend crucially on the periodicity of $\phi$. In this work, we propose and analyze a system, where such an observation-induced change of periodicity of the rotation angle $\phi$ can be realized, and will manifest itself, among other features, in a change of the periodicity of the $\mathrm{AB}$ effect and in different temperature dependence of the heat capacity and other thermodynamic properties.

The system we consider consists of two onedimensional loops of length $L_{1}$ and $L_{2}$, see Fig. 11. The first loop encloses a magnetic flux $\Phi$ and contains $N_{1}$ identical particles of mass $m_{1}$ and charge $q$ separated by an average distance $L_{1} / N_{1} \equiv a$. The $N_{2}$ particles in the second loop are also identical, with mass $m_{2}$, charge $q^{\prime}$, and average distance $L_{2} / N_{2} \equiv b$. We assume that all the particles are either spin-polarized or spinless, and do not

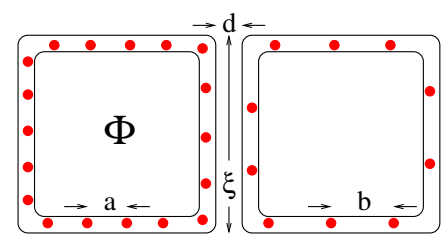

FIG. 1: Schematic of the system: two one-dimensional loops carrying Wigner molecules with average interparticle distances $a$ and $b$ that are approximately integer multiples of each other, $b \approx n a$; in the diagram, $n=2$. The two loops are separated by a distance $d$ that fulfills the condition $d \ll \xi$, where $\xi$ is the length of the interaction region. The first loop is threaded by a magnetic flux $\Phi$, while the second plays the role of a counter of the number of rotations in the first.

have internal degrees of freedom.

The idea is to study the quantum properties of the first loop in the regime when the second loop acts effectively as a detector counting the number of rotations performed by the particles in the first loop. This is achieved by assuming a strong Coulomb repulsion such that the particles in each loop form Wigner molecules (see, e.g., 15 17 and references therein). If the loops are sufficiently close to each other, the two Wigner molecules can be strongly coupled, as is known from studies of one-dimensional Coulomb drag [18 20]. The coupling is strongest if the particle densities in the two loops are approximately integer multiples of each other, $b / a \approx n$, see Fig. 1 which qualitatively depicts the case $n=2$. In general, a strong coupling between Wigner molecules allows to count the number of rotations of the first molecule in different ways, depending on the precise dynamics of the second one. In the regime of interest in this work, when $n>1$, and the second molecule is confined to a loop, the number of rotations is counted modulo $n$.

The $\mathrm{AB}$ effect provides the most direct manifestation of the periodicity properties of quantum dynamics. We start our analysis with a summary of this effect in one isolated loop. For strong Coulomb repulsion in the 1D setting, direct exchanges of particles are suppressed making the dynamics independent of the particle exchange statistics. It is therefore convenient to express the loop Hamiltonian in first-quantized notation in terms of the 
particle coordinates $x_{j}$ along the loop, and the corresponding momenta $p_{j}, j=1, \ldots, N$ :

$$
H_{0}=\frac{1}{2 m} \sum_{j=1}^{N}\left(p_{j}-q A\right)^{2}+\frac{1}{2} \sum_{i, j=1}^{N} U\left(x_{i}, x_{j}\right) .
$$

Here, $U \simeq q^{2} /\left(4 \pi \varepsilon \varepsilon_{0} r\right)$ is the Coulomb repulsion expressed in terms of $x_{j}$, and $A$ is the vector potential, which can be taken as a constant directly related to the AB flux $\Phi$ through the loop, $A=\Phi / L$. Depending on the geometry of the loop, $U$ can be a function of the two coordinates $x_{j}$ separately and not just of their difference. The results of this work do not depend on the precise form of the potential $U$, and are insensitive to deviations of the interaction potential in the real structure from the ideal Coulomb repulsion in a uniform medium.

We first assume that the loop is a circle. Then, by symmetry, $U\left(x_{i}, x_{j}\right)=U\left(x_{i}-x_{j}\right)$. For strong repulsion

$$
a \gg \varepsilon \varepsilon_{0} h^{2} /\left(m q^{2}\right),
$$

where $h$ is Planck's constant, the particles form a uniform Wigner molecule with equally-spaced equilibrium positions, $x_{j}=j a$. Defining the small deviations $u_{j}=$ $x_{j}-j a$, we can, as usual, expand the potential $U$ to quadratic order in $u_{j}$. The Hamiltonian (1) diagonalizes then in the Fourier coordinates $q_{l}$ and the conjugate momenta $p_{l}$,

$$
q_{l}=\frac{1}{N} \sum_{j=1}^{N} u_{j} e^{i 2 \pi j l / N}, \quad p_{l}=\sum_{j=1}^{N} p_{j} e^{-i 2 \pi j l / N},
$$

$l=0,1, \ldots, N-1$, and takes the form:

$$
\begin{aligned}
& H_{0}=\frac{1}{2 m N}\left(p_{0}-N q A\right)^{2} \\
& +\sum_{l=1}^{N-1}\left[\frac{p_{l} p_{N-l}}{2 m N}+N q_{l} q_{N-l} \sum_{j} U^{\prime \prime}(a j) \sin ^{2}\left(\frac{\pi l j}{N}\right)\right] .
\end{aligned}
$$

The second term (5) in this Hamiltonian describes $N-1$ normal modes of the Wigner molecule. An estimate of their frequencies $\omega_{l}$ shows that the condition (2) of strong interaction ensures that their energies $\hbar \omega_{l}$ are much larger that the energies $\epsilon_{i}$ of the center-of-mass (c.m.) motion of the Wigner molecule described by the first term (4). Thus, there exists a low-energy regime, when all normal modes are in the ground state, and the Hamiltonian $H_{0}$ reduces to the kinetic energy (4) of one particle of mass $N m$ and charge $N q$. The energies $\epsilon_{i}$ of this particle depend on the periodicity of the c.m. coordinate $y_{0}$. To determine the periodicity, note that a shift of the Wigner molecule by $a$ produces a particle configuration which can also be obtained by successive pairwise exchanges of one of the particles of the molecule with the other $N-1$ particles. This means that the wavefunction $\psi\left(y_{0}\right)$ of the c.m. motion satisfies the condition

$$
\psi\left(y_{0}+a\right)=\psi\left(y_{0}\right) e^{i \pi \nu(N-1)}
$$

where $\nu$ is the exchange statistics parameter of the particles in the loop, i.e., $\nu=0$ for bosons, $\nu=1$ for fermions, and a fractional $\nu$ for abelian anyons, which in principle can be controlled in the way discussed above in antidot structures in the fractional quantum Hall regime 2123$]$. Equation (6) shows that the c.m. coordinate effectively rotates as one particle on a circle with circumference $a$, with one full rotation corresponding to the one-period shift of the Wigner molecule. It also shows that the particle statistics affects only the phase, and not the amplitude or the period $\Phi_{0}$ of the $\mathrm{AB}$ oscillations. Indeed, a gauge transformation changing the quasiperiodic boundary condition (6) into a periodic one $\psi\left(y_{0}+a\right)=\psi\left(y_{0}\right)$, changes only the vector potential in the Hamiltonian $H_{0}$ (4) by adding the term $-\Phi_{0} \nu(N-1) / 2$, where $\Phi_{0}=h / q$ is the flux quantum for particles with charge $q$, to the flux $\Phi=A L$ through the loop. Then, the energies $\epsilon_{i}$ and the persistent current $I$ at low temperatures are

$$
\begin{aligned}
\epsilon_{i} & =\frac{N h^{2}}{2 m L^{2}}(i-\varphi)^{2}, \quad \varphi \equiv \frac{\Phi}{\Phi_{0}}-\frac{\nu(N-1)}{2}, \\
I & =\frac{N q h}{m L^{2}} \sum_{i} w_{i}(i-\varphi)
\end{aligned}
$$

where $w_{i}=e^{-\epsilon_{i} / T} / Z$ are the equilibrium Boltzmann probabilities. In term of the modified dimensionless flux $\varphi$, these expressions are independent of the statistics parameter $\nu$. For fermions, both the zero-temperature magnitude of the current $I$ (8), and the dependence of the phase of its oscillations on the parity of the number of particles $N$ 24], coincide with the case of non-interacting particles.

Note that Eqs. (7) and (8) remain valid for non-circular loops and in the presence of smooth disorder which does not pin down the Wigner molecule. In this non-ideal case, the particles are no longer uniformly spaced. This affects the spectrum of the normal modes of the molecule, but not the properties of the c.m. motion. Indeed, shifting the non-uniform molecule, $x_{j} \rightarrow x_{j+1}$, still changes the c.m. coordinate $y_{0}$ by $a=L / N$, since $N$ equivalent shifts clearly change $y_{0}$ by $L$, leaving the boundary condition (6) unchanged.

We now turn to the main focus of this work, viz., the properties of the $\mathrm{AB}$ oscillations for two coupled loops (Fig. 1). We consider the low-energy regime, when each loop acts as one particle that represents the c.m. motion. With the c.m. coordinates for the two loops denoted as $x$ and $y$, the Hamiltonian $H$ reads

$$
H_{0}=\frac{\left(p_{x}-N_{1} q A\right)^{2}}{2 M_{1}}+\frac{\left(p_{y}-N_{2} q^{\prime} A^{\prime}\right)^{2}}{2 M_{2}}+V(x-y),
$$

where $M_{j}=m_{j} N_{j}$ is the mass of the $j$ th Wigner molecule, $j=1,2$; the potential $V$ describes the interaction between the two loops, and we have added an $\mathrm{AB}$ flux $\Phi^{\prime}=L_{2} A^{\prime}$ through the second loop. Here and below we assume that the statistical contributions have already 
been included appropriately into the fluxes, as in Eq. (7), for both loops. For a sufficiently long and uniform interaction region, the potential $V$ depends only on the difference of the c.m. coordinates. A shift of either of the two molecules by one particle returns the system to the same configuration, implying that $V$ is periodic with period $a$. Since the system wavefunctions should have the period $a$ in $x$ and $b=n a$ in $y$, there are $n$ inequivalent minima of $V$. For the Coulomb interaction, and assuming that the length $\xi$ of the interaction region and the distance $d$ between the loops satisfy the condition $\xi \gg d \geq a$, this potential can be estimated as

$$
V(x-y)=V_{1} \cos \left[\frac{2 \pi(x-y)}{a}\right], \quad V_{1} \simeq \frac{\xi q q^{\prime} e^{-2 \pi d / a}}{2 \pi n a \varepsilon \varepsilon_{0} \sqrt{a d}} .
$$

Qualitatively, the basic physics of this model is that the large potential $V$ imposes the constraint $x=y$ (up to some constant) by suppressing tunneling between its $n$ inequivalent minima. The second loop acts then as a detector counting the number of rotations in the first loop modulo $n$, thus making the states of the first loop that differ by less than $n$ full rotations inequivalent. Thus, the periodicity of the c.m. coordinate $x$ increases from $a$ to $b=n a$, changing the energy spectrum, and reducing the period of the $\mathrm{AB}$ oscillations from $\Phi_{0}$ to $\Phi_{0} / n$.

To study the transition between the two periods quantitatively, we note that in relative and c.m. coordinates, $r=x-y, R=\left(x M_{1}+y M_{2}\right) / M_{\Sigma}$, where $M_{\Sigma} \equiv$ $M_{1}+M_{2}$, and the corresponding conjugate momenta, $p=\left(p_{x} M_{2}-p_{y} M_{1}\right) / M_{\Sigma}, P=p_{x}+p_{y}$, Eq. (9) separates into two independent terms, $H_{0}=H_{R}+H_{r}$ :

$$
\begin{aligned}
H_{R} & =\frac{1}{2 M_{\Sigma}}\left(P-N_{1} q A-N_{2} q^{\prime} A^{\prime}\right)^{2}, \\
H_{r} & =\frac{1}{2 \mu}\left(p-\frac{M_{2}}{M_{\Sigma}} N_{1} q A+\frac{M_{1}}{M_{\Sigma}} N_{2} q^{\prime} A^{\prime}\right)^{2}+V(r),
\end{aligned}
$$

where $\mu=M_{1} M_{2} / M_{\Sigma}$ is the reduced mass. In terms of the plane-wave solutions for $H_{R}$, and the Bloch functions $u_{s, k}(r)$, where $u_{s, k}(r+a)=u_{s, k}(r)$, for $V(r)$, the stationary states of $H_{0}$ can be written as

$$
\psi=e^{i(\kappa R+k r)} u_{s, k}(r),
$$

where the wavevectors $\kappa, k$ are determined by the boundary conditions which depend in general on the $\mathrm{AB}$ phases. These conditions are more conveniently stated in the c.m. variables $x, y$, and read, once the statistical contribution is included in the fluxes (cf. the single-loop case):

$$
\psi(x, y)=\psi(x+a, y)=\psi(x, y+n a) .
$$

Imposing these conditions on $\psi(10)$ we find the wavevectors $\kappa, k$ in terms of the wavevectors for $x$ and $y$ :

$$
\begin{array}{r}
\kappa=2 \pi\left(n_{y}+n n_{x}\right) / n a \equiv 2 \pi i / n a, \\
k=\frac{2 \pi}{n a M_{\Sigma}}\left(M_{2} n n_{x}-M_{1} n_{y}\right)=\frac{2 \pi n_{x}}{a}-\frac{2 \pi M_{1} i}{n a M_{\Sigma}},
\end{array}
$$

with integer $n_{x}, n_{y}$. The eigenenergies of $H_{0}$ can then be expressed in terms of the energy bands $\epsilon_{s}(k)$ in the periodic potential $V(r)$ :

$$
\begin{aligned}
\epsilon(i, s) & =\frac{h^{2}}{2 M_{\Sigma} a^{2} n^{2}}\left(i-\varphi^{\prime}-n \varphi\right)^{2} \\
& +\epsilon_{s}\left(\frac{2 \pi}{a n M_{\Sigma}}\left[M_{2} n \varphi+M_{1}\left(i-\varphi^{\prime}\right)\right]\right),
\end{aligned}
$$

where $\varphi$ and $\varphi^{\prime}$ are the fluxes through the two loops normalized to the corresponding flux quanta [cf. Eq. (7)], and we took into account that the energy bands $\epsilon_{s}(k)$ are periodic in $k$ with period $2 \pi / a$.

Equation (11) describes the transition between the two periods of the energy spectrum of the system in the magnetic flux $\Phi=\Phi_{0} \varphi$ through the first loop. For vanishing coupling potential $V$, the energy bands are given by the dispersion relation of a free particle, $\epsilon_{s}(k) \rightarrow(h k / 2 \pi)^{2} / 2 \mu$, and Eq. (11) reduces to the sum of the energies of two independent loops,

$$
\epsilon\left(n_{x}, n_{y}\right)=\frac{h^{2}}{2 M_{1} a^{2}}\left(n_{x}-\varphi\right)^{2}+\frac{h^{2}}{2 M_{2} n^{2} a^{2}}\left(n_{y}-\varphi^{\prime}\right)^{2},
$$

leading to the standard period $\Phi_{0}$ of the $\mathrm{AB}$ oscillations in flux $\Phi$. The periodicity in the flux $\Phi^{\prime}$ through the second loop as described by Eq. (11), is given by the flux quantum $\Phi_{0}^{\prime}$ of that loop regardless of $V$, consistent with our qualitative picture that the loop coupling does not change the periodicity of the quantum dynamics of the loop with the larger period. The periodicity in $\Phi$, however, changes if the loops are strongly coupled, roughly when $V_{1} 2 a^{2} \mu / h^{2} \gtrsim 1$. In this case, the energy bands become flat, $\epsilon_{s}(k)=$ const, and the energy of the system reduces to the first term in Eq. (11). A change of $\varphi$ by $\pm 1 / n$ in this term can be compensated by changing $i$ by \pm 1 , restoring the energy spectrum $\epsilon(i, s)$ to the initial form, while smaller changes of $\varphi$ modify the spectrum. Thus, the period of the spectrum $\epsilon(i, s)$ in the flux $\Phi$, and the resulting period of the $\mathrm{AB}$ oscillations of the persistent current $I$ is $\Phi_{0} / n$. Below, we take $\Phi^{\prime}=0$.

Figure 2 shows the persistent current $I$ in the first loop calculated using Eq. (11) as $I=$ $-\sum_{i, s} w(i, s) \partial \epsilon(i, s) / \partial \Phi$, where $w(i, s)$ are the Boltzmann probabilities. The plot illustrates the change of the $\mathrm{AB}$ oscillation period from $\Phi_{0}$ in the uncoupled regime, to $\Phi_{0} / n$ in the strongly-coupled regime, with increasing coupling strength. In the strongly-coupled case, the two Wigner molecules move together in a "phase-locked" way. For the mass ratio $M_{2} / M_{1}=0.1$ chosen in Fig. 2, the strong coupling does not change the mass of the moving object, $M_{1} \simeq M_{\Sigma}$, and all changes of the current, the period and the amplitude, are related to the change in periodicity of the first loop from $a$ to $n a$. Such a change of the period implies not only the reduction of the flux period by the factor $1 / n$, but also a similar reduction of the current amplitude, see Eq. (11) and Fig. 2. 


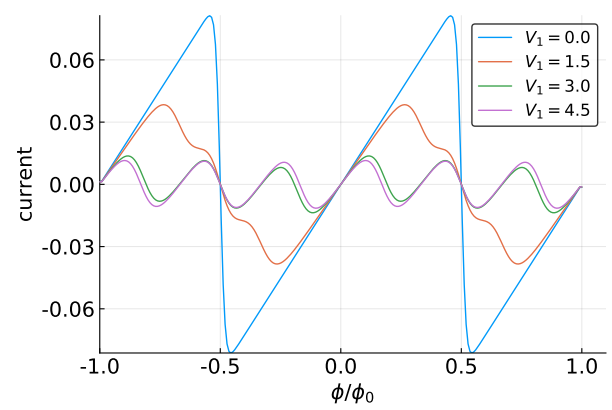

FIG. 2: Persistent currents for $n=3$ as a function of flux for different values of the dimensionless interloop coupling $V_{1} 2 a^{2} \mu / h^{2}$, temperature $k_{B} T=0.02 h^{2} /\left(2 a^{2} M_{\Sigma}\right)$, and mass ratio $M_{2} / M_{1}=0.1$. The flux period of the current changes from $\Phi_{0}$ for weak coupling to $\Phi_{0} / 3$ in the strong-coupling limit.

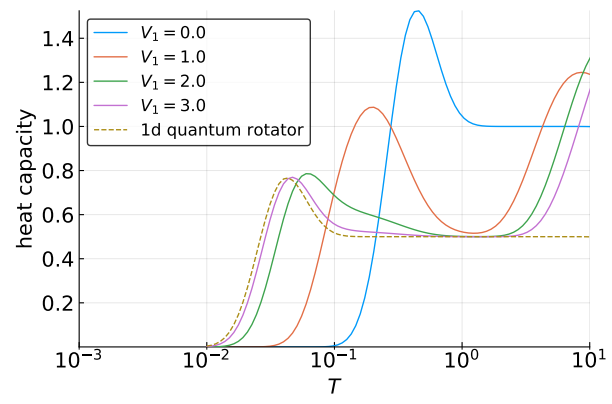

FIG. 3: Heat capacity in units of $k_{B}$ for different values of the dimensionless interloop coupling (see the caption of Fig. 2). The temperature is measured in units of $h^{2} /\left(k_{B} 2 a^{2} M_{\Sigma}\right)$.

The change of the spectrum described above has consequences for all observable properties. As an example, Fig. 3 shows the temperature dependence of the heat capacity for $\Phi=0$. For the parameters used in the plots, $n=3$ and $M_{2} / M_{1}=0.1$, the energy gaps in the spectrum of the two loops are nearly the same, $\Delta_{1}=h^{2} / 2 M_{1} a^{2} \simeq \Delta_{2}=h^{2} /\left(2 M_{2} a^{2} n^{2}\right)$, and without coupling, one gets the heat capacity $C$ of two nearly identical 1D quantum rotators. For each rotator, $C$ approaches $k_{B} / 2$ at large temperatures, and reaches a maximum of $C \sim 0.77 k_{B}$ at $T \simeq 0.38 \Delta$, see Fig. 3 At strong interloop coupling and low temperatures, the system turns effectively into one quantum rotator with a different period of rotation, and a different energy gap $\Delta=h^{2} /\left(2 M_{\Sigma} a^{2} n^{2}\right)$. Correspondingly, the heat capacity approaches the behavior of a 1D quantum rotator as shown in Fig. 3 .

The discussion above assumed an integer ratio of the periods of the two Wigner molecules, $b=n a$ with integer $n$. However, the main conclusion of our work on the $\mathrm{AB}$ period remains valid for $b=(n+\delta) a$, with finite $|\delta| \ll 1$. The proof of this statement consists of two steps. First, in the regime of strong interloop interaction $V$, the finite compression force $\partial U / \partial a$ in the Wigner molecules implies that for sufficiently small $\delta$, the two molecules will be compressed/expanded appropriately to make the relation between the periods precise in the interaction region. The interaction between the molecules will thus have the same qualitative features as in the ideal case: $n$ inequivalent minima of the periodic interaction potential. The second step of the proof relies on a geometric argument. Since even for the deformed molecules, as was argued above, the periodicities of their c.m. motion are given by $a$ and $b$, the configuration space of the coupled loops is a torus with circumferences $a$ and $b$. Geometrically, the precise reduction of the $\mathrm{AB}$ period by a factor $1 / n$ in the ideal case $\delta=0$ is a consequence of the fact that the trajectory following the minimum of the coupling potential $V$ makes $n$ turns around the $y$-axis of the torus, and thus encircles the flux $\Phi$ in the first loop $n$ times. As follows from the first step, this feature of the potential minimum, and with it, the $1 / n$ reduction of the $\mathrm{AB}$ period, remain valid even for $\delta \neq 0$, when the ratio of the two circumferences is not precisely integer.

The effects proposed here can in principle be observed in a variety of physical systems. One possibility is mesoscopic electronic structures which are sufficiently clean such that the Wigner-molecule physics used in our discussion is realized. Another possibility is arrays of Josephson junctions in the regime of individual Cooper-pair transport 25 29. Finally, arrays of trapped ions could also be used: translationally invariant ring-shaped ion traps with many ions have been experimentally realized [3032], and signatures of the $\mathrm{AB}$ effect in the ion dynamics have already been observed 33 .

To conclude, we have described a scenario in which the winding number of real-space rotations of a particle around a ring becomes a physical observable. The modified energy spectrum that results from the emergence of this observable leads to changes in the low-temperature behavior of quantities like the heat capacity and to a reduction in the Aharonov-Bohm period. An experimental realization of these effects will shed new light on the question of the indistinguishability of quantum states in interacting systems.

[1] L.S. Schulman, Techniques and applications of path integration (Wiley, New York, 1981), Ch. 23.

[2] R. Lynch, Phys. Rep. 256, 367 (1995).

[3] D.T. Pegg and S.M. Barnett, J. Mod. Opt. 44, 225 (1997).

[4] A. Luis and L.L. Sánchez-Soto, Prog. Opt. 41, 421 (2000).

[5] D.V. Averin, A.B. Zorin, and K.K. Likharev, Sov. Phys. JETP 61, 407 (1985).

[6] W. Zwerger, A.T. Dorsey, and M.P.A. Fisher, Phys. Rev. B 34, 6518 (1986).

[7] S.M. Apenko, Phys. Lett. A 142, 277 (1989).

[8] A. Davidson and P. Santhanam, Phys. Lett. A 149, 476 
(1990).

[9] D. Loss and K. Mullen, Phys. Rev. A 43, 2129 (1991).

[10] B.S. Deaver and W.M. Fairbank, Phys. Rev. Lett. 7, 43 (1961).

[11] W.A. Little and R.D. Parks, Phys. Rev. Lett. 9, 9 (1962).

[12] I.O. Kulik, JETP Lett. 11, 275 (1970).

[13] M. Büttiker, Y. Imry, and R. Landauer, Phys. Lett. A 96, 365 (1983).

[14] A.C. Bleszynski-Jayich, W.E. Shanks, B. Peaudecerf, E. Ginossar, F. von Oppen, L. Glazman, and J.G.E. Harris, Science 326, 272 (2009).

[15] K. Jauregui, W. Häusler and B. Kramer, Europhys. Lett. 24, 581 (1993).

[16] J.S. Meyer and K.A. Matveev, J. Phys.: Cond. Matt. 21, 023203 (2009).

[17] S. Pecker, F. Kuemmeth, A. Secchi, M. Rontani, D.C. Ralph, P.L. McEuen, and S. Ilani, Nat. Phys. 9, 576 (2013).

[18] K. Flensberg, Phys. Rev. Lett. 81, 184 (1998); Yu.V. Nazarov and D.V. Averin, ibid. 81, 653 (1998); V.V. Ponomarenko and D.V. Averin, ibid. 85, 4928 (2000).

[19] M. Yamamoto, M. Stopa, Y. Tokura, Y. Hirayama, and S. Tarucha, Science 313, 204 (2006).

[20] D. Laroche, G. Gervais, M.P. Lilly, and J.L. Reno, Science 343, 631 (2014).

[21] V.J. Goldman and B. Su, Science 267, 1010 (1995); I.J. Maasilta and V.J. Goldman, Phys. Rev. Lett. 84, 1776 (2000); V.J. Goldman, J. Liu, and A. Zaslavsky, Phys.
Rev. B 71, 153303 (2005).

[22] D.V. Averin and J.A. Nesteroff, Phys. Rev. Lett. 99, 096801 (2007).

[23] A. Kou, C.M. Marcus, L.N. Pfeiffer, and K.W. West, Phys. Rev. Lett. 108, 256803 (2012).

[24] D. Loss, Phys. Rev. Lett. 69, 343 (1992).

[25] Y. Nakamura, Yu.A. Pashkin, and J.S. Tsai, Nature 398, 786 (1999).

[26] V.E. Manucharyan, J. Koch, L.I. Glazman and M.H. Devoret, Science 326, 113 (2009).

[27] M.T. Bell, I.A. Sadovskyy, L.B. Ioffe, A.Yu. Kitaev, and M.E. Gershenson, Phys. Rev. Lett. 109, 137003 (2012).

[28] I.M. Pop, B. Doucot, L. Ioffe, I. Protopopov, F. Lecocq, I. Matei, O. Buisson, and W. Guichard, Phys. Rev. B 85, 094503 (2012).

[29] D.J. van Woerkom, A. Geresdi, and L.P. Kouwenhoven, Nat. Phys. 11, 547 (2015).

[30] R. Blatt and D. Wineland, Nature 453, 1008 (2008).

[31] B. Tabakov, F. Benito, M. Blain, C.R. Clark, S. Clark, R.A. Haltli, P. Maunz, J.D. Sterk, C. Tigges, and D. Stick, Phys. Rev. Applied 4, 031001 (2015).

[32] H.-K. Li, E. Urban, C. Noel, A. Chuang, Y. Xia, A. Ransford, B. Hemmerling, Y. Wang, T. Li, H. Häffner, and X. Zhang, Phys. Rev. Lett. 118, 053001 (2017).

[33] A. Noguchi, Y. Shikano, K. Toyoda, and S. Urabe, Nat. Comm. 5, 3868 (2014). 